\title{
The Known and Unknown of Mankind at the Times of Globalization
}

\author{
Yue Yang \\ Guangzhou, China \\ E-mail: yyllqq2007@163.com
}

\begin{abstract}
One of prominent traits for the time of globalization is information inflation and knowledge explosion. On one hand, human knows the nature more agreeably and the knowledge of nature tends to be more specific. Most are known. On the other hand, human understand the society more contrarily and the knowledge of society tends to be more confusing. Most are unknown. When and even before we are engaged in solving these globalization issues, it is extremely necessary to review and sequence human known knowledge and hope-to-know knowledge. In this paper, the author makes a comparative analysis of the unbalanced development of two kinds of knowledge systems at the time of globalization, and proposes a wish: promote human progresses and create a beautiful future by driving the balanced development of two kinds of knowledge systems.
\end{abstract}

Keywords: Globalization, Knowledge, System, Comparative analysis

Knowledge is the crystallization of human wisdom and can enrich human wisdom, the product of human reason and can enhance human reason. In an academic view, politics and laws are in the scope of humanism and society. If politics and laws are compared to two fruits, the knowledge system is the tree with more fruits; if compared to two trees, the forest with more trees; if compared to two forests, the land fostering wider forests ...... Here, from a larger point of view, we will make a macro observation over the knowledge system background of politics and laws.

Human wisdom and reason have experienced gradual-but-devious exploration and development for several thousands of years. Human abstracts amounts of knowledge from reasonable observation and thinking. With accumulated knowledge, the knowledge system comes into being after passing integration and reengineering. We can categorize the knowledge system by different angles. If we start from distinguishing the mutual relations between cognitive subjects and objects, knowledge system is divided into two groups: the first is the knowledge of nature that is founded on the relations of human and nature; the second is the knowledge of humanism and society that is founded on the relations of men.

One of prominent traits for the time of globalization is information inflation and knowledge explosion. On one hand, human knows the nature more agreeably and the knowledge of nature tends to be more specific. Most are known. On the other hand, human understand the society more contrarily and the knowledge of society tends to be more confusing. Most are unknown. When and even before we are engaged in solving these globalization issues, it is extremely necessary to review and sequence human known knowledge and hope-to-know knowledge.

\section{The great progresses and achievements of knowledge of nature}

Among all elements in an appropriate list, scientific knowledge is the right one that affects human history most prominently, profoundly, and strongly till now. The pushing effects of scientific knowledge exerted on the world and the earth where human are living during the process of huge changes, what are incomparable or irreplaceable by any other element. Human history is completely different in the centuries with undeveloped science or with developed science. "Today science enjoys a super fame. Undoubtedly, most are derived from the amazing achievements and the fast expansion of science in practice." (Carl G. Hemepel, 2006, p3)

Human has created science and has impressed by its powers and energies, so that human has built their hopes for a better future on science. To some extent, at least in some people's mind, science plays the role of savior of mankind. Since the day when there was human being, it is the science that gives human the initiative right of survival in nature, it is the science that helps human know natural phenomena and master natural laws, and it is also the science that drives human to change the nature to large scale and use the nature wisdom and abilities. "Science radically changes people's idea about their position in the nature. If human beings were expelled from the theater center created the universe, their arrogant attitudes will be suppressed. However, coming with the scientific revolution, it is that an unprecedented optimism to self abilities rushes into the minds of human beings. (Harman, P. M., 2003)" Except God, the greatest wisdom and power come from science. Do not be superstitious to science, but hold faith in science and trust science.

The epoch-making effect generated by natural scientific knowledge is obvious and well-known. Since the modern industrial revolution, energies released from the science reactors turn into a huge productivity, which completely 
changes the face of the world. Based on mastering natural science and knowing natural laws, human are engaged in production activities that take advantages of the nature and change the nature at an unprecedented depth and breadth. Thus, human regard the science as an omnipotent magic. The myth of science appears. People are in an illusion that they can access the developed and satisfying ideal society under the guidance of science. In fact, it is not the truth that science creates myth but science itself is myth. "Science is present myth, while myth was a scientific theory years ago. (P. K. Feyerabend, 2006)" However, during the two world wars occurred in succession in $20^{\text {th }}$ century, scientific technologies were used to produce advanced killing weapons, and could not do anything to kinds of social contradictions and conflicts. The myth of omnipotent science was shattered. Questions on the position and functions of science were there.

What on earth are reasons for the ups and downs of myth of science? Tracing the source, there are two types of scientific knowledge. They have different bases and at different development levels. On one hand, knowledge of nature has been booming, to be universally accepted, giving people powers, confidence, truth, and hopes. On the other hand, knowledge of humanities and society has lagged behind, to be universally diversified, making people frustrating, anxious, infighting, and disappointing. Two keys are for unlocking the locks. Each key is only to unlock a specific lock. With the brilliant achievements in knowing knowledge of nature, human tend to use scientific knowledge to solve all issues in front of mankind. But there is the dilemma. Knowledge of nature, as the key, can not unlock the lock of humanities and society. The key for knowledge of humanities and society is not in form or effective. Because of lagged-behind development of knowledge of humanities and society, all kinds of contradictions and conflicts at all levels within human society in all aspects can not be coordinated or negotiated rationally, and the integrated interests, objectives, and decisions for all human beings in the world can not be supported or established rationally. Thus, partial abuses of natural knowledge happen, and the negative effects hurt human beings. Therefore, the reason for negative effect of science is not the possible bad attributes of scientific knowledge but the unbalanced development of two types of knowledge. It is just the unbalanced, or seriously-unbalanced, development of two types of knowledge that results in human facing weirder opportunities and graver crises.

Unfortunately, human do not realize the unbalanced development of two types of knowledge. However, it is this imbalance that has led to the meaningless "science war" in the $90 \mathrm{~s}$ in $20^{\text {th }}$ century. It was actually quite intense, crowded, and widely spread. But in fact, science is also a kind of humanities. Humanities should be consistent with science. Scientific spirit is also a kind of humanities spirit, and humanities spirit should be consistent with scientific spirit. The opposition and conflict of the two are not only unnecessary waste of words, but totally blind, confused, and almost absurd.

\section{The lagged-behind development of social knowledge}

"SCIENCE has achieved great success in interpreting the world, which makes people admire it to a great degree. As a result, other theoretical systems can not help but want to dip in the light of science, so a part of humanities subjects are known as 'social science'. And the original SCIENCE is renamed as 'natural science'. (Song Tian, 2007, p36)" However, if we take the development course of natural science as a frame of reference, the development of science of humanities and society is far away from the level of Newton's era, probably still close to the era of Archimedes. Viewing the history, every branch of natural science has developed gradually from the lower level to the higher, from the backward stage to the advanced. The development course is clear and easy to notice. The comparable differences between the early development and the latest development are obvious. In contrast, no significant, solid, and convincing evidence shows that modern human philosophy, politics, ethics, laws, education, strategies, logics, and languages can be better than that during the Warring States period in China and during the time of ancient Greek and ancient Roman in the west. In other words, the development of every branch of humanities and society science does not form a course from the lower level to the higher, from the backward stage to the advanced. It is hard to compare its developments of the early and the later, or identify the differences between them.

Two signs indicate the development and the maturity of natural science today. First, people's recognition to the existence, the nature, and the laws of objective things in nature are deepening and growing, and more comprehensive. Second, people are more powerful in changing objective things in nature according to self recognitions and subjective wills that pursue for interests and avoid losses. Using natural science to explain the world, it is clear and consistent; using natural science to change the world, it is powerful and effective. In contrast, science of humanities and society is still very immature. If the level of development of natural science compared to the higher mathematics, the level of development of humanities and society science is still hovering around the elementary mathematics. In the field of humanities and society, it is impossible to say how much this doctrine exceeds that doctrine in accordance with universal standards. Science of humanities and society is still at a lower level. Confused concepts, random methods, different stances, amounts of schools, and inconvincible theories, all 
these conditions are far from real scientific norms and orders. "In the real sense, to development science of humanities and society, we mean to change the world but not only to explain the world. (Alan Chalmers, 2007,p22)" Unfortunately, using science of humanities and society to explain the world is ambiguous and confusing; and even using it to change the world is weak and inefficient. So far, all scientific revolutions occurred in human history are limited to the field of natural science. In the field of humanities and society science, no brilliant achievement has ever reached. It is still at the eve of scientific revolution.

"Historian Collingwood said: humans have a much stronger power of controlling the nature than two thousand years ago, but there is no ethical progress at all. (Song Tian, 2007, p139)" That is far from the facts. Today's development of humanities and society science does not go beyond the development of two thousand years ago when Chinese Confucius, Lao Zi, and Zhuang $\mathrm{Zi}$ and ancient Greek Socrates, Plato, and Aristotle have lived. One sign is that people's recognition to the existence, the nature, and the laws of society is not deepening or progressing. The other sign is that people do not strengthen the power of changing objective things in society in accordance with self recognition and subjective wills that pursue for interests and avoid losses. Cases illustrated this point can be found in any country of the world. Modern people are universally unsatisfied with social systems and social formation. People's descriptions of social systems and social formations are inconsistent. They can not explain the design of social systems and social formations properly. They feel helpless to the defects of social systems and social formations. And they are powerless to improve the social systems and social formations.

Take the relation between human and nature as the measure. Due to the development of natural science, human can master their fate more and more initiatively, and they can improve their living conditions more and more effectively. The science of nature has created a broader and smoother path for engineering technology. Humans use the science of nature to understand the nature more deeply. At the same time, they use engineering technologies to change the nature at a large scale. Agricultural technologies can significantly increase the crop yields to feed more people. Industrial technologies can supply more and better production tools and daily necessities. Medical technologies can prevent and cure more human diseases. Architect technologies can build higher and practical houses, factories, and commercial buildings. Transportation technologies can provide faster and more convenient transport services. Communication technologies can help people actualize more timely communication and connection. Cyber technologies can enable people to access richer browsing information.

Take the relation between humans as the measure. Because of immaturity of humanities and social sciences, human can not actively control their own destiny or change their living conditions effectively. Social conflicts and problems become more complicated, more serious, and more confusing. With common perceptions, modern people do not feel better social fairness and justice today than ever, or better social safety then ever, or more happiness than ever. To be rational, no any discipline of scholar can prove that today's social fairness and justice are better than ever, today's social safety is better than ever, or today's happiness is more than ever.

In every discipline of natural science, all scholars are in mutual cooperation basically. For these scholars, there is either a parallel-and-interactive relation or a before-and-after successive relation. The building of theories of one discipline is constructed with the joint effects of different scholars. In constructing the same building of theories, some scholars lay a foundation, some supply backbones, and some add bricks. On the contrary, in every discipline of humanities and social sciences, all scholars are work by themselves basically. For different scholars, there is either a parallel-and-adversarial relation or a before-and-after negative relation. They are not cooperating together to build the building of theories of one discipline. A school or even a scholar just builds their own huts or bunkers. Therefore, for each discipline, there are numerous independent huts or bunkers in the field of each discipline but not any real common theoretical building. That is the distinct difference between humanities and social sciences, and natural sciences.

In areas of natural sciences, laws named after surnames of scholars and theoretical models named after some abstract concepts are numerous. These laws and theories are connected and complemented mutually, like a ladder with thousands of steps or a delicate machine with amounts of parts, forming the organic disciplinary system in each special field. In contrast, although kinds of doctrines named after surnames of scholars or abstract concepts are also numerous in areas of humanities and social sciences, just like in areas of natural sciences, these doctrines are either overlapped or mutually negative, unlike steps forming a ladder, or parts forming a machine, forming different disciplinary kingdom in each specific field, just like the separation in feudal society.

For state of knowledge, "science is rigorous, systematic, and organized knowledge. Science has the following traits distinguishing it from other knowledge: close to no contradiction (internal harmony); can be verified; can be repeated; with predictability. (Biwen Cao, et al, 2006, p1)" Moreover, "the systematic knowledge generated by scientific activities is logical, empirical, rational, rigorous, and universal. (Biwen Cao, et al, 2006, p319)" These 
attributes are only consistent with natural sciences but not humanities and social sciences.

In areas of natural sciences, truth is dominating, no place for lies or nonsense. In areas of humanities and social sciences, truth is struggling, and all places are right for the prosperity of lies and nonsense.

In areas of natural sciences, truth is universally acknowledged, belonging to all human beings as the consensus of humans and the common assets shared by all humans. In areas of humanities and social sciences, truth is personal, belonging to different schools, nations, parties, and countries, being the separated assets labeling by schools, nations, parties, and countries, standing for their ownership.

In areas of natural sciences, truth is truth, and can not be faked by preaching, posters, slogans, ideas, vows, and promises. In areas of humanities and social sciences, truth is a mixed bag, and can be faked by preaching, posters, slogans, ideas, vows, and promises.

In areas of natural sciences, truth is supreme, and can not be suppressed or distorted by powers or violence. In areas of humanities and social sciences, truth is a humble, and can be suppressed or distorted by powers or violence.

In areas of natural sciences, truth has the attributes of purity and toughness, and can not be corrupted or confused by tricks. In areas of humanities and social sciences, truth is mixed and fragile, and can be corrupted or confused by tricks.

In short, confronting with nature, human knowledge is rising, powers from knowledge are strengthening, efficiency of using knowledge is improving, and wealth created by knowledge is growing. Confronting with society, human resentments are rising, crimes are growing, sufferings are increasing, and so do disasters. If we want to change the contrast, we should summarize inspirations and references from the development of natural sciences, select the new directions, and design new paths, triggering out the vitality of humanities and social sciences, and driving the fast development of humanities and social sciences.

\section{Promote the balanced development of natural knowledge and social knowledge}

At present, globalization has led human to a fork in a road, connecting with history and future. For the future, there are two directions. One is the better road, and the other is the crisis. In walk forward the better road, we must overcome obstacles; if walk forward the crisis road, we just let it happen. Except science, nothing can help human beings make the correct choice in the fork in a road. However, we have to firstly solve the issue of knowledge balance in order to make science generate sufficient effects. Therefore, humans should strive for the balanced development of two scientific systems, making it replace the useless conflicts between scientific culture and humanities culture.

The coordinative and balanced development of scientific system means: on one hand, natural science will keep in developing and progressing indefinitely; on the other hand, the scientific pattern will change from the one that the development of humanities and social sciences lagged behind the development of natural sciences to the one that two scientific systems develop equally. During the process of changes, an integrated reaction between the two scientific systems will happen: the true scientific spirits of natural sciences will be developed in areas of humanities and social sciences; the true scientific methods of natural sciences will be spread and practiced in areas of humanities and social sciences; the true scientific concepts of natural sciences will be taken as inspirations and references in areas of humanities and social sciences.

For the relation between man and nature, we should perform the scientific targeting of objectives, and build a harmonious relation instead of the conquered one. It is unscientific to say that human conquer the nature. Human do not necessarily conquer the nature. And they are incapable of conquering the nature. The relation between man and nature should not be the conquering-and-conquered one. In dealing with the relation between man and nature, the objective is to know the nature as much as possible, make best use of nature rationally, and change the nature properly. "Conquering the nature" is an irrational arrogance of human beings.

For the relation between human beings, we should also perform the scientific targeting of objectives, and build a harmonious relation to get rid of the intense relation. The original relation between human beings is either harmonious or disharmonious. Everyone should initiatively improve a harmonious relation with others, avoiding, reducing, and eliminating a disharmonious relation. The disputes and conflicts between people cover the fields of understandings and interests. Ignorance, prejudice, and misunderstandings are prevalent in all levels, corners, and places of the society, and easy to be spread. They serve as important reasons for triggering, stimulating, and deepening a disharmonious relation between people. Science contributes a lot to the construction of a harmonious relation between people since it has an active and effective effect on correcting ignorance, overcoming prejudice, and clarifying misunderstandings. "The possibility of science improving human happiness is not merely limited to the decrease of 'evil' things in human nature. How much science contributes to the better and active elements are 
maybe endless. (Russell, B., 2007, p326)"

Considering the fact that social contradictions and cultural conflicts are endangering the survival and happiness of human more and more, today what we need is to make scientific research on society and culture instead of scientific social and cultural research. Unfortunately, people just go in the opposite direction. Hope that this situation can be corrected as soon as possible, turning to a scientific research on society and culture, and speeding up the progress of humanities and social sciences. The ultimate objective is to supply powerful scientific support for a beneficial development of society and culture.

Knowledge is power. The well-known words of Bacon are widely circulated in the world and have been cited numerous times. However, compared with the strong powers generated by natural sciences, the powers of humanities and social sciences are still weak (to a great degree, it is caused by the oppositions of different forces). The powers generated respectively by two scientific systems should be influencing in human progresses. Just as medical knowledge can effectively help people diagnose, treat, and prevent various physical illnesses, knowledge of humanities and social sciences can effectively help people to identify, correct, and overcome various human weaknesses. Just as knowledge of system science and relevant disciplines can help people to follow the established goals, take reasonable strategies, mobilize the necessary resources, optimize the design schemes, produce various products, and accomplish different projects, knowledge of humanities and social sciences can help people to follow the established goals, take reasonable strategies, mobilize the necessary resources, optimize the design schemes, create various institutions, and actualize different ideals. However, it is still unpredictable for when and where the God send a genius, just like Newton, to the areas of humanities and social sciences.

Anybody is qualified and has reason to ask: why could not we exclude and prevent wars, poverty, and drugs, like excluding and preventing cholera, typhoid, and malaria? Why could not we improve the level of morals and spiritual civilization greatly, like improving the quantity of crops and the speed of transports? Areas of humanities and social sciences demand for new scientific enlightenments and discoveries. Reviewing the history of natural science, from Claudius Ptolemaeus' geocentric theory to Nicolas Copernicus' heliocentric theory, from Galileo's law of free fall to Newton's laws of motion, from Democritus' atomic theory to John Dalton's atomic theory, from Boyle's concepts of chemical elements to Mendeleev's periodic table of elements, from Linnaeus' biological system classification to Darwin's theory of evolution, from Coulomb's law on the charge to Ohm's law on the current, from Thomson's atomic structure model to Rutherford's, from Michelson-Morley's “ether" drift experiment to Einstein's theory of relativity, from de Broglie wave to Schrodinger equation ...... it shows us a picture: a number of outstanding scientists are running in a relay for exploring the secrets of the nature. They inherit from their ancestors and develop further. With their hard-word, the recognition of human to objective laws of nature is deepening and improving. People hope to see the same scene in areas of humanities and social sciences, leaving all new presentations and new packages for changeable words and terms of various doctrines and theories as history, inspiring new scientific discoveries, and making people's recognition to objective laws in areas of humanities and social sciences deepening and improving continuously.

"So far, the greatest impact on our daily lives is from natural science. But in the future, the impact of physiology and psychology will be more. When we find how a character depends on physiological situation, we can produce amounts of desirable man as we like. Wisdom, aesthetic ability, and benevolence ------ all these can be undoubtedly increased because of science. As long as people can use the science with wisdom, it will be endless for people doing for creating a beautiful world. (Russell, B., 2007, p326)" The wisdom and energy of science can help people to advocate objectiveness and fair, fairness and reasonability, selecting the best and discarding the worst, taking interests and avoiding hurts, closing kindness and punishing evils, in people's recognition, decision, and action. Science has played a role in history and will continue to play a role in: exploring the secrets of world, developing fields of knowledge, exploring and using resources, creating human miracles, changing the face of nature, promoting social development, guide civil progress, creating a prosperity of culture, strengthening communication and connection, improving understanding and consensus, expanding life welfare, and benefiting healthy and vitality. Science has no boundaries. It is the common cause and asset of humanity. Science can surpass all cultural barriers and become the main motive and channel for trans-cultural communication.

"Once we recognize that understanding is a means of establishing knowledge, we can run through from natural sciences to humanities and social sciences. (Micheal Ploanyi, 2004, p163)" Though this run- through needs a long process, in a new era it will come true. The scientific revolution done in areas of natural sciences must advance areas of humanities and social sciences. When scientific revolution develops well and gains successes in areas of humanities and social sciences, when the development of humanities and social sciences catch up with natural sciences at the same height and maturity, when academic researches in areas of humanities and social sciences are democratic, liberate, and equal, humans will learn how to treat and handle the problems facing them with rational 
attitudes and thinking, making scientific analyses and recognitions to the nature of personal and social problems, and making scientific evaluations and decisions on solutions for personal and social problems. In scientific prospect, the goal of progress in human history and the track should be: taking wisdom, knowledge, strength, and wealth as growing neutral independent variables, the negative value function (resentment, guilt, threat, misery, disaster, and fear) declines along with the rise of neutral independent variables (wisdom, knowledge, strength, and wealth) in proportion; the positive value function (harmony, kindness, tolerance, security, justice, and happiness) rises along with the rise of neutral independent variables (wisdom, knowledge, strength, and wealth) in proportion.

\section{Reference}

Alan Chalmers. Translated by Jiang, Jinsong. (2007). Science and Its Fabrication. Shanghai: Shanghai Science \& Technology Education Press. Aug. p22.

Cao, Biwen. Liu, Lixia \& Jia, Zhan. (2006). Introduction to Science and Technology. Beijing: Publishing House of Electronics Industry. Aug. p1, 319.

Carl G. Hemepel. Translated by Zhang, Huaxia. (2006). Philosophy of Natural Science. Beijing: China Renmin University Press. Nov. p3.

Harman, P. M. Translated by Zhiye. (2003). The Scientific Revolution. Shanghai: Shanghai Translation Publishing House. Dec.

Micheal Ploanyi. Translated by Wang, Jinghua. (2004). Science, Faith, and Society. Nanjing: Nanjing University Press. Jun. p163.

P. K. Feyerabend. Et al. Translated by Jian, Chen, et al. (2006). Knowledge, Science, and Relativism. Nanjing: Jiangsu People's Publishing House. Jan.

Russell, B. translated by Guo, Yigui. Et al. Russell on Freedom. Beijing: World Affairs Press. Jan. p326.

Tian, Song. Edited by Jiang, Xiaoyuan. (2007). Strategies and competition for scientific word right. Look! Scientism. Shanghai: Shanghai Jiaotong University Press. Jan. p36, 139. 